\title{
Mobile Health Technologies for Suicide Prevention: Feature Review and Recommendations for Use in Clinical Care
}

David D. Luxton, Ph. D., M.S. ${ }^{1, *}$ Jennifer D. June, M.S. ${ }^{2}$ Samantha A. Chalker, B.A. ${ }^{1}$

\author{
Address \\ ${ }^{*}, 1$ Department of Psychiatry and Behavioral Sciences, University of Washington \\ School of Medicine, Seattle, Box 356560, Seattle, WA, 98195, USA \\ Email: ddluxton@uw.edu \\ ${ }^{2}$ National Center for Telehealth \& Technology, Tacoma, WA, USA
}

Published online: 26 September 2015

(C) Springer International Publishing AG 2015

This article is part of the Topical Collection on Suicide

Keywords mHealth · Mobile applications $\cdot$ Apps $\cdot$ Technologies $\cdot$ Suicide $\cdot$ Self-directed violence

\section{Opinion statement}

Mobile devices, and the apps (software applications) that operate on them, have potential to help manage and prevent suicidal behavior by assisting with assessment of risk, providing educational and support information, and facilitating access to safety plans, crisis support, and coping tools. This clinician-friendly review provides an overview of the principal features of currently available mobile health apps that are specially designed for suicide prevention. The use of patient contact interventions (i.e., text messaging and email interventions) on mobile devices is also discussed. Recommendations for selecting and integrating these tools into patient care are provided, along with discussion of emerging capabilities and limitations including the lack of research regarding the clinical effectiveness of mobile health apps. The topics presented should be informative to individual practitioners as well as health care organizations that are considering adopting these technologies into their services.

\section{Introduction}

Over the last decade, we have witnessed the rise of the smartphone and advances in tablet computers that have placed powerful point-of-use tools into the hands of both patients and health care professionals. The growth and popularity of mHealth apps (health-related software applications) are staggering; by 2018, it is estimated that $50 \%$ of the more than 3.4 billion smartphone and tablet users will have downloaded 
mHealth apps [1]. Mobile devices, and the apps that operate on them, provide highly accessible and convenient capabilities for self-care, clinical assessment, clinical decision-making, and treatment delivery. Mobile technologies also provide a platform for contact interventions (e.g., SMS texting), scheduling and appointment reminders, and the capability for synchronous

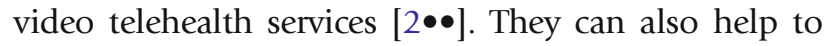
address some of the most difficult challenges associated with suicide prevention including the need for better methods for the assessment of risk, facilitating access to crisis support and coping resources, creating and accessing safety plans, and providing support information for patients and family members.

With the growing quantity of mobile applications available and their potential to help with suicide prevention, it is advantageous for health care providers to be familiar with what is available to them and their patients and to know how to appropriately integrate these tools into care. Our goals with this paper are therefore to provide a clinician-friendly review of the principal features of available mHealth apps that are designed to help with prevention of suicide and self-directed violence and to discuss need-to-know considerations for selecting and integrating these technologies into clinical practice. We primarily focus on apps that are available for free and produced by reputable government and nonprofit organizations in the USA. There are also many apps designed to address mental health conditions, such as depression, that may include suicide prevention features. Although these are not reviewed here, we recommend that readers see the reviews by

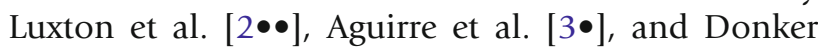

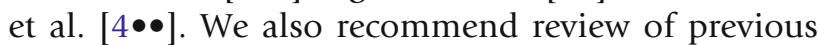
publications on the topic of technologies for suicide prevention by Luxton, June, and Kinn [5] and the edited volume by Mishara and Kerkhof [6•].

\section{Key features of mobile applications for suicide prevention}

The majority of existing suicide prevention apps share multiple common features and functions, which are often integrated into a single app. In real-world application, clinicians and their patients may find that just one or a few of the features of an app are useful or needed at any given time. In this section, we discuss these principal features and functions of suicide prevention apps. A list of suicide-prevention-specific apps and their features is provided in Table 1.

\section{Information, education, and training}

Most of the suicide prevention apps that are available include education functions that provide information to patients, care providers, and other individuals (i.e., peers and family members). This feature typically entails lists of facts about suicide, narratives, and also web links to additional information and resources. For example, SuicideSafe, developed by the Substance Abuse and Mental Health Services Administration (SAMHSA), provides information support to those who are at risk for suicide as well as for health care providers. The app is based on Suicide Prevention Resource Center's (SPRC) physical pocket card Suicide Assessment Five-Step Evaluation and Triage (SAFE-T) [7] and is intended to help providers understand the SAFE-T approach. The app also provides case studies examples, fact sheets, best-practice tips for talking to patients, and ways to find local treatment resources, and like many other suicide-prevention-associated apps, it provides information about available crisis lines. 


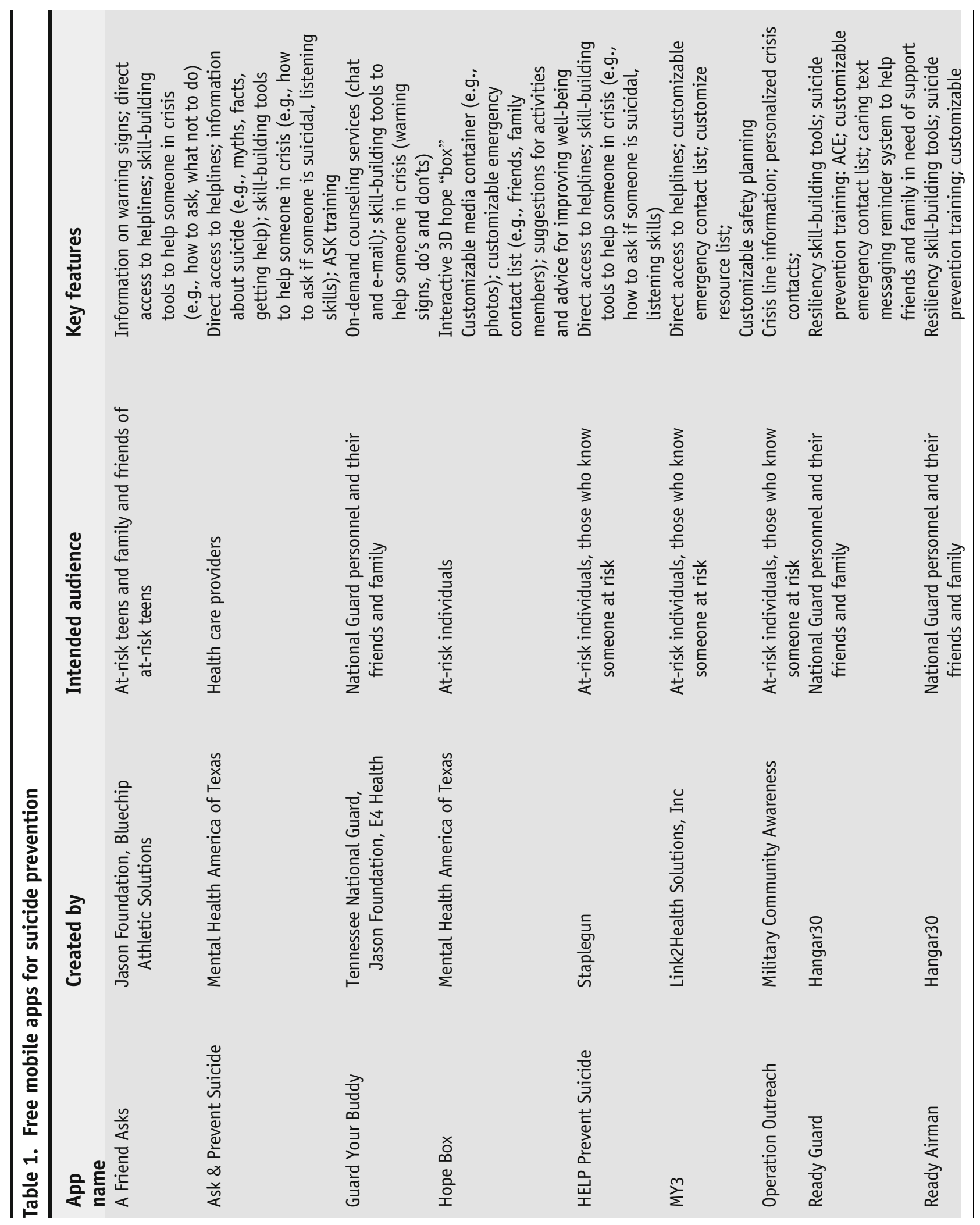




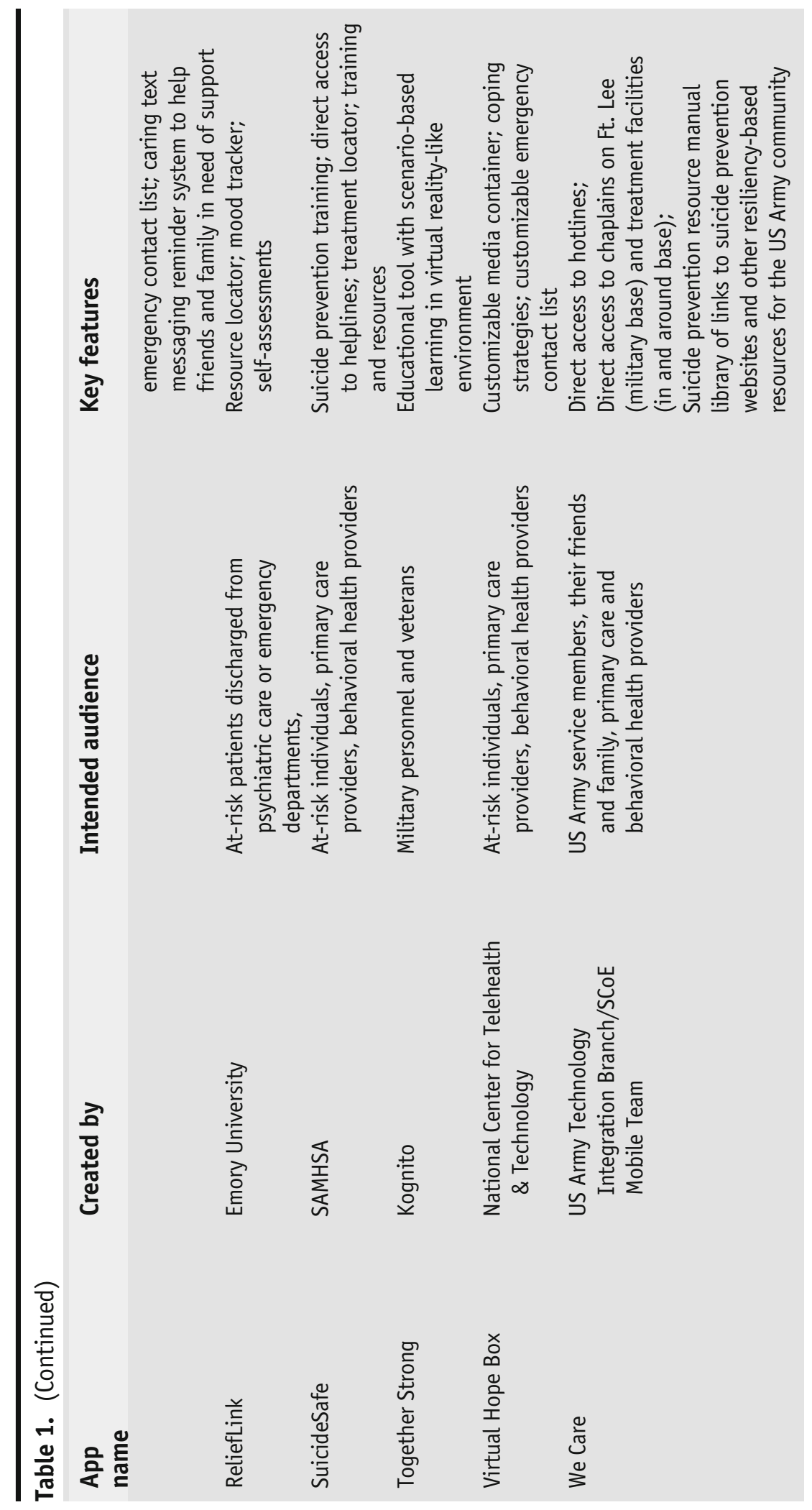


Mobile device apps can also provide help resource location such as crisis support centers, medical facilities, or groups or individuals (e.g., peers, family members) who could assist and provide support. The "emergency button" (or "hot-button" or "red-button") feature makes use of the mobile device's cellular phone, e-mail, or internet connection for one touch direct connection to a crisis line or other resources whenever and wherever a person may feel in crisis.

The award winning ReliefLink app is one example of an app with resource location and emergency button features [8]. The app is intended to help facilitate continuity of care and follow-up care linkages for patients following discharge from an inpatient unit or emergency department. The app makes use of a map feature and the global positioning (GPS) function available on most smartphones to locate resources that are in proximity to the user. ReliefLink also includes mood and behavior monitoring and tracking features, a safety-planning feature, medication and appointment reminders, built-in coping tools (i.e., relaxation and mindfulness exercise, relaxing radio music), and an emergency button that can connect patients to helplines, health care providers, 911, and friends/family members [8]. Another example is the MY3 app (www.my3app.org) that was created in partnership with Link2Health (the administrator of the National Suicide Prevention Lifeline) and the California Mental Services Authority with funding provided by the California Mental Health Services Act (Prop. 63). This app allows persons with history of suicidal behavior or who have been experiencing suicidal thoughts to create a support network of three main contacts that they would normally go to in times of need and stress. Contacts can also include a provider and or a crisis center, and all of which may be accessed via the app.

Resource location and emergency button features are particularly useful considering that a patient may need help in off-hours. Users may also appreciate the simplicity and ease-of-use of the emergency button feature and the peace-of-mind of knowing that it is there if needed. Family members or other persons when supporting a person in crisis may also appreciate the emergency button and resource location features. We suspect that some health care providers may be concerned about patients overusing the emergency button feature in lieu of other coping strategies. We did not find any evidence of this issue reported in the literature.

\section{Safety planning and other coping tools}

Mobile apps provide for easily accessible and more interactive safety plans. The aforementioned $M \gamma 3$ app, for example, provides a template to create a safety plan that is adapted from content developed by the US Department of Veterans Affairs and Stanley and Brown [9]. Information about how to use the app that is available on the MY3 website encourages individuals to first write down their plan on paper before input into the app, and the instructions also advise that it is best to work with clinicians when developing their safety plan.

Other apps are designed to provide coping tools that may be used to augment traditional treatments. The Virtual Hope Box (VHB), developed at the 
National Center for Telehealth and Technology (US Department of Defense), is based on the analog version of the "hope box" concept, a therapeutic tool used by clinicians to help depressed and/or suicidal patients redirect their negative thoughts to reflect on reasons for living [10, 11]. A traditional hope box consists of a collection of personal items, such as personal accomplishments (e.g., recognition and achievement awards), family photos, and favorite music or art that serve to lift patients' spirits when they are feeling down or struggling with life's pressures [10-12]. The VHB provides a similar container function by allowing patients' to use their own phones to add personalized content such as pictures, video, and music for immediate access when they are feeling distressed. The VHB also features positive coping messages, mindfulness exercises, and activity planning content [13].

\section{Clinical assessment and automated intervention}

Mobile devices provide a convenient platform for conducting assessment with

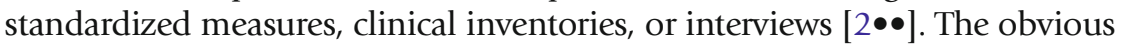
advantage of assessment via mobile devices is $24 / 7$ access as well the potential to share the data with health care providers. The inclusion of electronic versions of measures and surveys on mobile devices also allows the resulting data to be integrated with other functions of the app, such display of data on graphs to track change over time or to direct users to review a safety plan or to contact help resources. For example, the ReliefLink app includes a "mood tracker" tool that includes assessment of suicidal thoughts. Users can indicate their current state by selecting from a series of "weather-based" icons that are connected to text responses. An icon of the sun, for example, indicates "no suicidal thoughts" whereas an icon of a thunderstorm indicates "Strong user to act on the plan immediately." If a user selects a response indicating suicidal thoughts, a pop-up message appears at the bottom of the screen that states, "Feeling low? Help is within reach!" When the pop-up message is selected, a window appears with a list of resource contact options such as for calling the National Suicide Prevention Lifeline, access to the customized safety plan, or other resources to help the user find a health care provider or support group nearby.

Mobile technologies also provide opportunities for sophisticated data collection methods for the purposes of detecting risk in real-time and directing interventions [14•]. Specifically, mobile devices and wearables (e.g., smart watches) can be used to collect data for ecological momentary assessment (EMA). EMA involves the sampling of current behaviors and experiences in real time in the natural environment [15]. Predictive analytics entails extracting information from existing data sets in order to determine patterns and to predict emerging (future) outcomes [16]. Multi-modal data inputs may include Internet and social media usage (i.e., social activity), surveys taken on a mobile device (i.e., symptom data), behavioral data (e.g., exercise and sleep pattern data collected by smart watches), and environmental data (e.g., location near places associated with risk). If automated analysis of the data detects heightened risk, the system could automatically alert health care providers and/or the user to use their safety plan and coping skills. One example of this type of system for suicide risk detection is The Durkheim Project [17]. The Durkheim Project began as a Defense Advanced Research Projects Agency (DARPA)-sponsored 
project that established and validated risk metrics for suicide risk and a capability to scale to larger participant "opt-in" networks. Essentially, the project involved the collection of data from various inputs (e.g., smartphone and social media use), which is processed and then sent into an analytics environment via a secure communication network for analysis. The Durkheim Project team is currently exploring the use of this predictive capability for advancing suicide risk detection and intervention for US military veterans [17].

\section{Mobile devices and patient contact}

Mobile technologies also provide opportunities for suicide prevention interventions that include contact with patients via the use of texting, e-mail, and telephone features that are available on most mobile devices. Some of the uses of texting and e-mail in health care are to send treatment appointment reminders and behavioral reminders and to provide individual-level support [18]. The use of this technology may be especially useful for addressing suicide risk among patients following treatment, including after release from psychiatric inpatient units or emergency departments [19].

One suicide prevention intervention that makes use of e-mail, texting, postal mail, or phone contact with patients is the "Caring Contacts" intervention [19]. The intervention is based on the earlier "caring letters" work of Jerome Motto and colleagues $[20,21]$ which showed that psychiatric inpatients who refused follow-up care but were sent brief letters from hospital staff that espoused caring concern had a significantly lower rate of suicide in the first 2 years after discharge than did patients who did not receive the letters. The intervention is purported to exert its suicide preventative affect through the maintenance of a caring connection between care providers and high-risk persons. The contacts may also serve as reminders of available treatment and provide an avenue for the patient to reconnect to care (i.e., by replying to the messages or making use of links to suicide prevention and counseling hotlines provided in the contacts). Further, follow-up contacts may help patients to feel better about treatment and therefore motivate them to seek or adhere to treatment [19].

Both texting [22-24] and e-mail [25] interventions for suicide prevention have been previously evaluated, but randomized controlled trials are needed. Comtois and colleagues at the University of Washington are presently conducting a trial that is investigating the use of text messaging for suicide prevention at US military outpatient behavioral health clinics and inpatient facilities [26]. Initial data from the trial has supported the feasibility of posttreatment texting contacts, and the participants' evaluations of the caring text messages were very positive [27]. Luxton and colleagues are presently testing caring e-mails to prevent suicide among US military personnel and veterans [28]. In both the aforementioned trials, patient replies to the messages have not been excessive and all crisis replies were managed successfully by following established safety protocols $[27,28]$. Whether or not any of the aforementioned contact interventions that involve patient contact through electronic mediums will be feasible in your practice setting will depend on local regulations regarding posttreatment contact with patients, including use of these electronic mediums for patient contact (for more information about caring contacts methodology and implementation considerations, see Luxton, June, and Comtois [19] and Luxton et al. [28]). 


\section{Table 2. Checklist for selecting and integrating mHealth apps for use in clinical care}

\begin{tabular}{|c|c|}
\hline Step & Specific considerations \\
\hline 1. Identify clinical needs & $\begin{array}{l}\text { - Determine what tool or application may best meet patient needs: } \\
\text { - Treatment } \\
\text { - Coaching/support } \\
\text { - Self-care } \\
\text { - Information only } \\
\text { - Determine patient preferences for use of the technological tool: } \\
\text { - Assess experience with technologies } \\
\text { - Determine access to technologies (i.e., smartphones) }\end{array}$ \\
\hline $\begin{array}{l}\text { 2. Determine what apps are available and assess } \\
\text { quality of the tool/application }\end{array}$ & $\begin{array}{l}\text { - Visit resources } \\
\text { - Search app stores } \\
\text { - Read available literature regarding mHealth apps } \\
\text { - Review published scientific literature } \\
\text { - Review user feedback } \\
\text { - Attend training } \\
\text { - Discuss with colleagues }\end{array}$ \\
\hline 3. Review regulatory and data security requirements & $\begin{array}{l}\text { - Does application meet HIPAA requirements } \\
\text { - Does application fall under medical device requirements? } \\
\text { - Review local policies } \\
\text { - Review safety risks }\end{array}$ \\
\hline 4. Evaluate/test it & $\begin{array}{l}\text { - Test it yourself before use } \\
\text { - Solicit feedback from patients }\end{array}$ \\
\hline 5. Plan use/develop protocols & $\begin{array}{l}\text { - Discuss safety/expectations for use with patient } \\
\text { - Include technology procedures/expectations in informed } \\
\text { consent procedures } \\
\text { - Plan protocols for use } \\
\text { - Review existing or } \\
\text { - Develop protocols (who, when, and how to use the application) }\end{array}$ \\
\hline
\end{tabular}

\section{Putting mobile apps to use in clinical care}

While our focus is on the use of these technologies for the treatment and management of suicidal patients in care and aftercare, the following recommendations are generalizable to all types of mHealth apps across health care settings. We also provide a checklist of recommended steps in Table 2.

\section{Identify clinical need and appropriateness}

An initial step should always be to assess what the individual patient needs and to match the appropriate intervention and tools to these needs. As we noted earlier, mobile apps are typically intended to augment clinical care, and thus, how they accomplish this may vary from one clinical situation (patient's needs) to another. For example, some patients may benefit solely from the informational component of a mobile app, whereas others may benefit from the resource location function of an app. It is also essential to assess the patient's preferences for using technology, their experience with technology, access to 
technology, or any other concerns that the patient may have (e.g., how any collected data may be used, security concerns). This information is important for determining fit between patient and technology as well as what additional training or coaching may be required to help the patient feel comfortable and confident with the technology. Clinicians should also be mindful of any cognitive deficits or other variables (e.g., inadequate vision) that may impair a patient's use of technology. If a patient finds that the app is not applicable to their needs, is too difficult to use, or they do not trust it, they will likely abandon use of it.

\section{Determine what apps are available and assess their quality}

Due to the large amount of apps that are available in the marketplace, it can be difficult for users (health care providers, patients, or others) to determine the best app for their needs and whether it will provide accurate information. Moreover, even if an app is advertised as "evidenced-based" because it consists of content from a tested therapeutic approach, it does not mean that use of the app is evidenced-based. Unfortunately, there is not an app certification process in place at present time that involves formal review of apps for appropriateness and effectiveness in any given application [29]. It is therefore essential to review available information about any app of interest to assess the overall quality and appropriateness. We hope that this review will provide a helpful starting point. We also recommend review of available app resources, such as the International Association for Suicide Prevention (IASP) website about suicide prevention and intervention apps (https://www.iasp.info/resources/Suicide_Prevention_and_ New_Technologies/Suicide_Prevention_Smartphone_Apps/).

We also encourage review of available published literature regarding the app or program involving its use as well as a search on the web for information from the manufacturer or organization that produced it. Some organizations provide user manuals or clinical guides that may be helpful. The app description on the app storefront (i.e., iTunes, Google Play, Amazon App Store for Android phones), user reviews, and the number of downloads also provide indicators of the user friendliness and quality of the app. We also recommend discussing apps with colleagues and attending training workshops or conference proceedings regarding the topic.

\section{Regulatory, data security, and policy review}

The responsible use of mobile apps during clinical practice also requires that practitioners and health care organizations to be cognizant of privacy risks and overall data security requirements. Protected health information (PHI) of users can be stored on these devices and transmitted over networks to and from health care providers. As noted by Luxton, Kayl, and Mishkind [30•], mHealth security threats are present during both storage and transmission of electronic patient data and can jeopardize patient data as well as the integrity of entire data networks. Furthermore, adherence to applicable laws and regulations associated with patient privacy and safety is essential for ethical and legal practice.

In general, patients may do whatever they choose with their own data that is collected, stored, or transmitted on mobile devices. However, if the patient transmits or shares any electronic PHI with a Health Insurance Portability and Accountability Act (HIPAA)-covered entity, then the health care professional becomes responsible for HIPAA compliance [2••]. In 2013, as part of the Health Information Technology for Economic Clinical Health (HITECH) Act, 
the penalties for HIPAA noncompliance have become more severe [31]. Readers may wish to review the American Medical Association's summary of the new rule available at http://www.ama-assn.org/ama/pub/physician-resources/ solutions-managing-your-practice/coding-billing-insurance/hipaahealthinsurance-portability-accountability-act.page?

The Food and Drug Administration (FDA) provides regulatory control and guidance regarding mobile devices that function as medical devices and defines them as a device "whose functionality could pose a risk to a patient's safety if the mobile app were not to function as intended" [32]. The FDA considers a medical device to be a product intended to prevent or treat any aspect of human functioning [33]. In order for a mHealth app to fall under current FDA regulation, it must meet this definition and be intended to be used as an accessory to an already regulated medical device or to transform a mobile platform into a regulated medical device [32]. The FDA specifies that not all mobile apps will meet these criteria, and it provides several examples of types of mobile apps that it does not intend to enforce, such as those that are education/training tools only or those that automate office operations. In general, most mental health apps, including all included in Table 1, will fall into the non-regulated category. In lieu of any formal accreditation/certification of mHealth apps, however, we recommend review of the FDA requirements and any documentation provided by the manufacturer of a mHealth app. Clinicians should always review any institutional-level policy regarding the use of these technologies.

Evaluate and test

We recommend that any app of interest be tested prior to use in clinical practice. Become familiar with all of its features and its limitations, and review information within the app to determine if it is accurate. Once you put the app to use, solicit regular feedback from your patients regarding their experiences with the app. This will help you to determine what may work best for your patient and whether non-technological alternatives may be more appropriate.

Safety protocols and expectations regarding use of the app should be discussed with patients prior to use. Specific topics to be discussed during the informed consent process and throughout care as needed should include expectations regarding contact via electronic means. Electronic forms of communication, such as e-mail and texting, are immediate, and thus, there may be an expectation by patients for immediate response [19]. Thus, expectations, including typical response times and limits of availability of health care providers, should be addressed. A plan for what happens if a patient loses their smartphone or if the battery runs out of power should also be discussed during the informed consent process.

Since all of the apps presented here are freely available to the public, patients may download and use them without collaboration with a clinician. It is thus a good idea to ask patients about their awareness of and use of apps or other resources, such as social media (which is accessed primary via mobile devices). As discussed by Luxton, June, and Fairall [34], social media can have both benefits and negative impact toward the prevention of suicide and self-directed 
violence. Social media can provide a place for peer support (suicide prevention and survivor support groups) and also a tool to link people to help resources. On the other hand, social media has been associated with cyber bullying or a source to learn about suicide methods. A review of patient's use of and experience with mHealth apps and social media can be helpful for determining additional resources and exposures to risks.

\section{Emerging capabilities with mobile technologies}

We can expect the future to bring further improvements in the integration of mobile health technologies, including the use of wearables [14•]. As we stated previously, wearable devices can be used to collect data in real time that can be combined with data from smartphones to provide rich data on context and behavior that may confer behavioral risks, such as self-directed violence. This technology could be integrated with an automated alert system as well as a caring text intervention that automatically sends appropriate behavioral reminders to patients. Also, Malott and colleagues [35] have conducted preliminary testing of the use of wearable devices to detect self-harming behaviors of inpatients. The system, called A Self-Harm Activity Recognition Engine (SHARE), infers self-harming activities (e.g., wrist cutting) by making use of accelerometer data from a smart watch worn on patient's wrists.

Another emerging technology with great promise is the application of virtual humans (also referred to as virtual artificial intelligent agents). Virtual humans are computer-generated characters that are capable of human-like verbal and nonverbal behaviors (i.e., natural language processing and expression of emotions, etc.) [36]. Virtual humans have been tested as an effective way to provide interactive training and education in medical settings, such as for training of behavioral health clinicians [36] and for providing hospital discharge planning to patients [37]. Virtual humans have also been tested on mobile devices as a means to provide counseling and coaching [14•], and the company Kognito has developed an app called Together Strong that is a mobile version of Family of Heroes, a role-playing simulation of a mental-health-based training program [38]. Users can engage in scenarios with a virtual human character to practice conversing with someone in crisis in order to improve listening skills, learn how to de-escalate arguments, and learn how to help motivate a person to seek help.

There are several limitations associated with mHealth technologies that must be considered when integrating them into care. First, not all patients will have access to these technologies. There are economic limitations as well as technological barriers (e.g., quality of telecommunications networks or access to electricity) that may limit access for some patients (e.g., the homeless) or entire communities and countries in the world. There is also a general lack of data regarding the clinical effectiveness of mHealth apps, including those designed for suicide prevention, as well as data from larger program evaluation that involve these apps. The scarcity of data regarding how well these apps have helped clinicians, raised awareness about the problem of suicide, or have connected people to care is also a limitation. As noted by de Beurs and colleagues [39•], most experimental studies rely on convenience samples, often students or patients from a nearby hospital, thereby limiting generalizability. 


\section{Conclusion}

What are sorely needed are randomized controlled trials that evaluate mobile technologies that augment traditional or other novel suicide prevention interventions.

In addition to these broader limitations, the adoption of mHealth technologies into care can sometimes be hindered in health care organizations due to a lack of awareness or knowledge of their capabilities, concerns about patient privacy (i.e., electronic data security), lack of supporting technology infrastructure, or economic barriers. The identification of a local "champion," who can help educate an organization and guide and train other clinicians, may be helpful.

Mobile technologies provide innovative tools that can help address some of the challenges associated with the prevention of suicide and self-directed violence. These tools are not intended to replace the skills of traditional health care providers, but to augment standard treatments and risk management approaches to suicide prevention. Suicide prevention apps also provide resources that are immediately available when they are needed and they have potential to enhance traditional therapeutic interventions by reinforcing coping behaviors and skills outside of the clinic setting. We are hopeful that through continued technological innovation, research, and integration of these technologies into care, these tools will further improve the care and safety of persons who are at risk for suicide.

\section{Compliance with ethics guidelines}

\section{Conflict of Interest}

David D. Luxton declares that he has no conflict of interest. Jennifer D. June declares that she has no conflict of interest. Samantha A. Chalker declares that she has no conflict of interest.

\section{Human and animal rights and informed consent}

This article includes review of studies conducted by one or more of the authors of the current paper, in which human subjects participated, but does not present new or unpublished data from these studies.

The opinions or assertions contained herein are the private views of the authors and are not to be construed as official or as reflecting the views of the Department of the Army or the Department of Defense.

\section{References and Recommended Reading}

Papers of particular interest, published recently, have been highlighted as:

- Of importance

$\bullet \quad$ Of major importance

1. Research2guidance. Mobile health market report 20132017: the commercialization of mHealth applications

(Vol. 3) 2013 Available at: http://www.research2guidance. com/shop/index.php/mhealth-report-2 
2.• Luxton DD, McCann RA, Bush NE, Mishkind MC, Reger GM. mHealth for mental health: integrating smartphone technology in behavioral healthcare. Prof Psychol: Res Pract. 2011;42:505-12. doi:10.1037/a0024485.

Provides general overview of mobile technologies in behavioral health care and clinical considerations.

3. $\quad$ Aguirre RT, McCoy MK, Roan M. Development guidelines from a study of suicide prevention mobile applications (apps). J Technol Hum Serv. 2013;31(3):26993.

Provides a review of suicide preventions apps developed by human service organizations (HSO).

4.• Donker T, Petrie K, Proudfoot J, Janine Clarke J, Birch M, Christensen H. Smartphones for smarter delivery of mental health programs: a systematic review. J Med Internet Res. 2013;15(11):e247. doi:10.2196/jmir. 2791.

Provides a systematic review of research supporting the efficacy of mental health-based mobile applications.

5. Luxton DD, June JD, Kinn JT. Technology-based suicide prevention: current applications and future directions. Telemed J E Health. 2011;17:50-4.

6. Mishara B, Kerkhof A. Suicide prevention and new technologies: evidence based practice. Palgrave Macmillan. 2013. Provides reviews of the application of various technologies that can assist with suicide prevention and risk management.

7. SAFE-T pocket card. U.S. Department of Health and Human Services Substance Abuse and Mental Health Services Administration. 2009. Available at: http:// www.sprc.org/library_resources/items/safe-t-pocketcard

8. Emory University, Woodruff Health Sciences Center. Suicide prevention app awarded $\$ 50,000$ prize at White House Conference. September 17, 2013. http:// news.emory.edu/stories/2013/09/kaslow_relieflink_ app/campus.html. Accessed 22 July 2015.

9. Stanley, B. \& Gregory K. Brown, G.K. Safety plan template. 2008. http://www.sprc.org/sites/sprc.org/files/ SafetyPlanTemplate.pdf. Accessed 5 Aug 2015.

10. Berk MS, Grosjean M, Warnick HD. Beyond threats: risk factors for suicide in borderline personality disorder. Curr Psychiatr. 2009;8:32-41.

11. Wenzel A, Brown GK, Beck AT. Cognitive therapy for suicidal patients: scientific and clinical applications. American Psychological Association. Washington: DC; 2009.

12. Berk MS, Henriques GR, Warman DM, Brown GK, Beck AT. A cognitive therapy intervention for suicide attempters: an overview of the treatment and case examples. Cogn Behav Pract. 2004;11:265-77.

13. National Center for Telehealth \& Technology. Virtual Hope Box. 2015 Available at: http://t2health.dcoe.mil/ apps/virtual-hope-box. Accessed 11 Aug 2015.

14. Luxton DD, June JD, Sano A, Bickmore T. Mobile, wearable, and ambient intelligence in behavioral health care. In: Luxton DD, editor. Artificial intelligence in behavioral and mental health care. New York: Elsevier/Academic Press; 2016. Presents mobile and wearable technologies for behavioral health with examples.

15. Moskowitz DS, Young SN. Ecological momentary assessment: what it is and why it is a method of the future in clinical psychopharmacology. J Psychiatry Neurosci. 2006;31(1):13.

16. Luxton DD, editor. Artificial intelligence in behavioral and mental health care. New York: Elsevier/Academic Press; 2016.

17. Poulin C, Thompson P, Bryan C. Public health surveillance: predictive analytics and Big data. In: Luxton DD, editor. Artificial intelligence in behavioral and mental health care. New York: Elsevier/Academic Press; 2016.

18. Free C, Phillips G, Galli L, Watson L, Felix L, Edwards P, et al. The effectiveness of mobile-health technologybased health behaviour change or disease management interventions for health care consumers: a systematic review. PLoS Med. 2013;10(1), e1001362. doi:10. 1371/journal.pmed.1001362.

19. Luxton DD, June JD, Comtois KA. Can post-discharge follow-up contacts prevent suicide and suicide behavior?: a review of the evidence. Crisis. 2013;34:32-41. doi:10.1027/0227-5910/a000158.

20. Motto JA. Suicide prevention for high-risk persons who refuse treatment. Suicide Life Threat Behav. 1976;6:223-30.

21. Motto JA, Bostrom AG. A randomized controlled trial of postcrisis suicide prevention. Psychiatr Serv. 2001;52:828-33.

22. Berrouiguet $S$, Alavi Z, Vaiva G, Courtet P, Baca-Garcia E, Vidailhet $P$, et al. SIAM (Suicide intervention assisted by messages): the development of post-acute crisis text messaging outreach for suicide prevention. BMC Psychiatry. 2014;18(14):294.

23. Berrouiguet S, Gravey M, Le Galudec M, Alavi Z, Walter $M$. Post-acute crisis text messaging outreach for suicide prevention: a pilot study. Psychiatry Res. 2014;217(3):154-7.

24. Chen H, Mishara BL, Liu XX. A pilot study of mobile telephone message interventions with suicide attempters in China. Crisis. 2010;31:109-12.

25. Luxton DD, Kinn JT, June JD, Pierre LW, Reger MA, Gahm GA. The caring letters project: a military suicide prevention pilot program. Crisis: J Crisis Interv Suicide Prev. 2012;33:5-12.

26. Comtois KA. Military Continuity Project (MCT): a suicide prevention study. In: ClinicalTrials.gov [Internet]. Bethesda (MD): National Library of Medicine (US). April 8, 2015. https://clinicaltrials.gov/ct2/show/ NCT01829620. Accessed 2 Aug 2015.

27. Comtois KA. Feasibility and acceptability of caring contacts via text: increasing connection with marines and soldiers to prevent suicide attempts and ideation. In D D Luxton (Chair), Post-treatment technologybased caring contacts for suicide prevention. 28th World Congress of the International Association for Suicide Prevention, Montreal, Canada, 2015. 
28. Luxton DD, Thomas EK, Chipps J, Relova RM, Brown D, McLay R, et al. Caring letters for suicide prevention: implementation of a multi-site randomized clinical trial in the U.S. military and Veteran Affairs healthcare systems. Contemp Clin Trials. 2014;37(2):252-60. doi:10.1016/j.cct.2014.01.007.

29. Powell AC, Landman AB, Bates DW. In search of a few good apps. JAMA. 2014;311(18):1851-2. doi:10. 1001/jama.2014.2564.

30. Luxton DD, Kayl RA, Mishkind MC. mHealth data security: the need for HIPAA-compliant standardization. Telemed e-Health. 2012;18:284-8. doi:10.1089/ tmj.2011.0180.

This article describes need-to-know data security requirements for mobile health devices.

31. U.S. Government Publishing Office. 78 FR 5565 Modification to the HIPAA privacy, security, enforcement, and breach notification rules under the Health Information Technology for Economic and Clinical Health Act and the Genetic Information Nondiscrimination Act; other medication to the HIPAA rules. January 25, 2013. http://www.gpo.gov/fdsys/granule/FR2013-01-25/2013-01073/content-detail.html Accessed 29 July 2015.

32. Food and Drug Administration. Mobile medical applications-guidance for industry and food and drug administration staff. February 9, 2015. http://www.fda. gov/downloads/MedicalDevices/

DeviceRegulationandGuidance/Guidanc eDocuments/ UCM263366.pdf. Accessed 2 Aug 2015.

33. U. S. Food and Drug Administration. What is a medical device? July 20, 2015. http://www.fda.gov/AboutFDA/
Transparency/Basics/ucm 211822.htm. Accessed: 29 July 2015.

34. Luxton DD, June JD, Fairall JM. Social media and suicide: a public health perspective. Am J Public Health Res. 2012;102(2):195-200. doi:10.2105/AJPH.2011. 300608.

35. Malott L, Bharti P, Hilbert N, Gopalakrishna G, Chellappan S. Detecting self-harming activities with wearable devices. In Pervasive Computing and Communication Workshops (PerCom Workshops), 2015 I.E. International Conference. 2015:597-602.

36. Rizzo AA, Lange B, Buckwalter JG, Forbell E, Kim J, Sagae K, et al. An intelligent virtual human system for providing healthcare information and support. Stud Health Technol Inform. 2011;163:503-9.

37. Bickmore T, Utami D, Barry B, Henault L, Waite K, Matsuyama, R, et al. Enabling web search for low health literacy individuals using conversational agents. Washington, DC: Health Literacy Annual Research Conference (HARC). 2013.

38. Albright G, Goldman R, Shockley KM, McDevitt F, Akabas S. Using an avatar-based simulation to train families to motivate veterans with post-deployment stress to seek help at the VA. Games Health J. 2012;1(1):21-8.

39.• de Beurs D, Kirtley O, Kerkhof A, Portzky G, O'Connor $\mathrm{RC}$. The role of mobile phone technology in understanding and preventing suicidal behavior. Crisis. 2015. Discusses current mobile technology as applied to suicide prevention. 